\title{
Archives of Clinical and Biomedical Research
}

Volume 1, Issue 3

Research Article

\section{A Retrospective Study on the Risk of Non-Melanoma Skin Cancer in PUVA and Narrowband UVB Treated Patients}

\section{Darukarnphut P, Rattanakaemakorn P*, Rajatanavin N}

Division of Dermatology, Faculty of Medicine, Ramathibodi Hospital, Mahidol University, Thailand

*Corresponding Author: Rattanakaemakorn P, Division of Dermatology, Faculty of Medicine, Ramathibodi Hospital, Mahidol University, Thailand, E-mail: ploysyne@gmail.com

Received: 04 April 2017; Accepted: 24 April 2017; Published: 28 April 2017

\begin{abstract}
Background: Narrow-band ultraviolet B (NB-UVB) and Psoralen-UVA (PUVA) phototherapy are widely used as the treatment of dermatologic diseases. The carcinogenic risk associated with NB-UVB and PUVA phototherapy treatment is unknown.

Objective: To determine the non-melanoma skin cancer risk in a patients treated with Narrow-band ultraviolet B (NB-UVB) and Psoralen-UVA (PUVA) phototherapy.

Methods: All Narrow-band ultraviolet B (NB-UVB) and Psoralen-UVA (PUVA) phototherapy treated patient records who received the phototherapy in Ramathibodi Hospital from 2001 to 2015 were reviewed.

Results: A total of 726 with Narrow-band ultraviolet B (NB-UVB) and Psoralen-UVA photochemotherapy (PUVA) phototherapy treated patients with mean age of 46.7 years. Most of patients have skin phototypes III to IV. Vitiligo is the most common disease treated with phototherapy (43.8\%). The median number of NB-UVB treatment was 126 treatments with cumulative dose $94.2 \mathrm{~J} / \mathrm{cm}^{2}$. The median number of PUVA treatment was 103 treatments with cumulative dose $249.2 \mathrm{~J} / \mathrm{cm}^{2}$. There was no non melanoma skin cancer in our patients. The median time of follow up was 28.4 months.
\end{abstract}


Conclusion: This study did not shown an increase risk of skin cancer in patients who had receiving long term NBUVB and PUVA phototherapy. We suggest that long term NB-UVB and PUVA phototherapy are safe therapeutic modalities for the patients with skin phototypes III to IV.

Keywords: Non Melanoma Skin Cancer Risk; NB-UVB and PUVA Phototherapy

\section{Introduction}

Psoralen-UVA photochemotherapy (PUVA) phototherapy and narrow-band ultraviolet B (NB-UVB) phototherapy are the effective treatments for dermatologic diseases such as psoriasis, atopic dermatitis, vitiligo, and mycosis fungoides [1]. Non-melanoma skin cancers (NMSC) are generally associated with cumulative exposure to ultraviolet radiation [2]. The incidence of non-melanoma skin cancers associated with phototherapy was limited. PUVA is associated with a definite cumulative exposure and UVA dose related increase in the risk of skin cancers [3,4]. There was no definite associations between NB-UVB phototherapy and skin cancer were found in retrospective studies carried out in Northern Ireland, Scotland and German, an increase risk of non-melanoma skin cancer among patients treated with both PUVA and NB-UVB was reported.

Whereas, the European studies focused on Caucasian patients and their findings may not be applicable to nonCaucasians [5,6,9]. PUVA and NB-UVB phototherapy are widely used in Thailand but the photocarcinogenic risk of PUVA and NB-UVB in patients with skin phototypes III-V was unknown. Our study investigated the occurrence of NMSC in a patients who were treated with PUVA and NB-UVB phototherapy.

\section{Material and methods}

\subsection{Patients}

The data analyzed in this retrospective study were collected from patients' medical records. Between January 2001 and December 2015, 956 patients with psoriasis, vitiligo, atopic dermatitis, livedoid vasculitis, pityriasis lichenoides chronica, and other dermatoses received PUVA, NB-UVB or both modalities at phototherapy clinic, Ramathibodi hospital. 230 patients who had either the follow up period less than two months or the total treatment sessions less than twenty were excluded. Most patients were treated two or three times per week. Treatment began with an initial dose of $50-70 \%$ of the minimal erythema dose (MED) followed by a 10-20\% incremental dose regimen. During phototherapy, the patients received routine facial and genital protection.

We reviewed patient medical records to obtain information on demographic characteristics, skin phototype, total number of phototherapy sessions, total cumulative dose, medical treatment combination phototherapy and other relevant with items. Reported non-melanoma cancer cases were classified according to the International Classification of Diseases for Oncology 3rd edition and converted according to the International Classification of Diseases 10th edition (ICD-10). 


\subsection{Statistical analysis}

Expected numbers of cases in the PUVA, NB-UVB and both modalities treated group and the standardized incidence ratios (SIRs) for NMSC were calculated from the incidence rates in the control population. Statistical analyses were performed using SPSS version 17.0.

\section{Results}

\subsection{Demographic Data}

Demographic data for the 726 patients who underwent phototherapy more than 20 sessions or follow up period more 2 months between January 2001 and December 2015 are shown in Table 1. The mean age was 47 years old. There was slightly female predominance (60\%). Ninety nine percent of the patients were Thai. 38 patients were classified as having skin type II, 360 skin type III, 185 skin type IV and skin type were not recorded for the remaining 143 patients. Of these 726 patients, the most common underlying diseases of the patients was vitiligo 318 (43.8\%) followed by psoriasis 269 (37.1\%), mycosis fungoides 59 (8.1\%), pityriasis lichenoides chronica 17 (2.3\%), hand and foot eczema 12 (1.7\%), atopic dermatitis 9 (1.2\%), livedoid vasculitis 9 (1.1\%) and others 34 (4.7\%) (Figure 1). The most common drug use combination with phototherapy was methotrexate 161 (22\%), followed by azathioprine 87 (12\%), cyclosporine 76 (11\%), acitretin 54 (7\%), and others 33 (5\%) (Figure 2). There was one patient with two lesions of basal cell carcinoma (BCC) on face and remission after surgical treatment. The skin cancer were developed before the initiation of phototherapy and no recurrence after long term follow up.

\begin{tabular}{|lc|}
\hline Parameters & Values \\
\hline Number of patients & 726 \\
Age, year, mean \pm SD & $46.7 \pm 16.2$ \\
Sex, N(\%) & \\
$\quad$ Female & $432(59.5)$ \\
Race, N(\%) & \\
$\quad$ Thai & $719(99.2)$ \\
Fitzpatrick's skin phototype, N(\%) & \\
$\quad$ II & $38(5.2)$ \\
$\quad$ III & $360(49.6)$ \\
$\quad$ IV & $185(25.5)$ \\
$\quad$ No record & $143(19.7)$ \\
Phototherapy, N(\%) & \\
$\quad$ PUVA & $33(4.5)$ \\
NB-UVB & $624(86)$ \\
Both PUVA and NB-UVB & $69(9.5)$ \\
Pre-existing skin cancer & 2 BCCs \\
& \\
\end{tabular}

Table 1: Demographic parameters in patients treated with phototherapy at Ramathibodi hospital 
SD: standard deviation

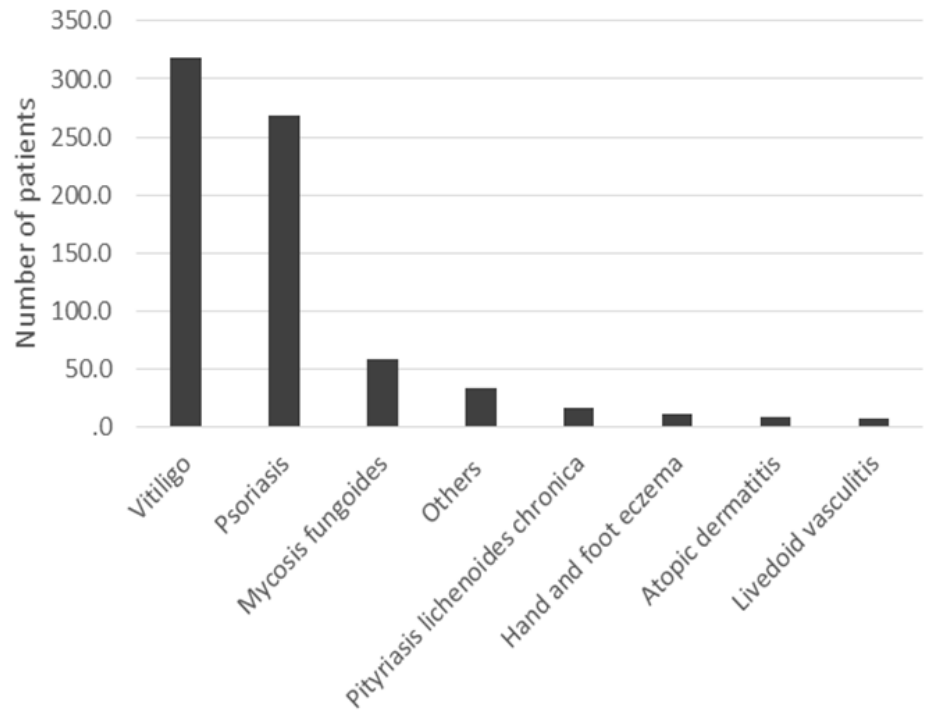

Figure 1: The underlying disease of the patients treated with phototherapy

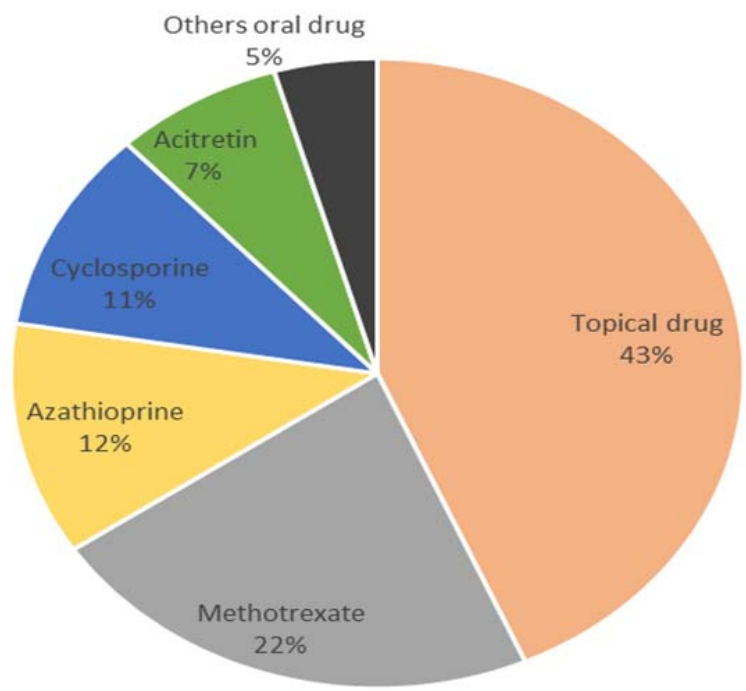

Figure 2: The combination drug use in the patients treated with phototherapy

\subsection{PUVA, NB-UVB and both modalities phototherapy}

There was 33 (4.5\%) patients treated with PUVA, 624 (86\%) treated with NB-UVB and 69 (9.5\%) treated with both modalities. The average number of phototherapy sessions in PUVA group was 103.3 and 126.3 in the NB-UVB. The average cumulative dose of PUVA was $249.2 \mathrm{~J} / \mathrm{cm}^{2}$ and $94.5 \mathrm{~J} / \mathrm{cm}^{2}$ in NB-UVB group. The average number of phototherapy sessions of PUVA and NB-UVB was 112 and 87, the average cumulative dose of PUVA and NBUVB was 360.3 and $146 \mathrm{~J} / \mathrm{cm}^{2}$ and the median time of follow up was 28.4 months in PUVA and NB-UVB group (Table 2). 


\begin{tabular}{|lcccc|}
\hline Parameters & PUVA & NB-UVB & \multicolumn{2}{c|}{ PUVA\&NB-UVB } \\
\hline Number of patients, & $33(4.5)$ & $624(86)$ & $69(9.5)$ \\
$\mathrm{N}$ (\%) & & & 28.4 \\
Time of follow-up, & 39.7 & 26.8 & $(2-264)$ \\
months, median (range) & $(2-244)$ & $(2-434)$ & PUVA & NB-UVB \\
& & & 360.3 & 146 \\
Cumulative dose, J/cm² & 249.2 & 94.5 & $(40.8-3795.2)$ & $(0.3-1530.8)$ \\
Median (range) & $(40.8-3795.2)$ & $(2.1-2059.7)$ & 122 & 87 \\
Number of sessions, & 103.3 & 126.3 & $(20-877)$ & $(6-579)$ \\
Median (range) & $(20-597)$ & $(20-877)$ & - & - \\
Number and types of & - & - & & \\
cancers observed & & & & \\
\end{tabular}

Table 2: Summary of the therapeutic parameters for PUVA, NB-UVB and both modalities

\subsection{Development of skin cancer}

There was no skin cancer after phototherapy in this study. There was one patient who developed two lesions of BCC on her nose and was treated eight months before the initiation of phototherapy, she received two-year-course of NBUVB for her psoriasis and there was no recurrence of skin cancer after five year follow up.

\section{Discussion}

In our retrospective study, the medical records of 956 patients treated with phototherapy were reviewed. Among the 726 whose records were analyzed. In this study, the most common underlying diseases was vitiligo, followed by psoriasis. The majority of participants belonged to Fitzpatrick skin phototype III and IV. The most common combination drug use with phototherapy was methotrexate, followed by azathioprine. There was no non-melanoma skin cancer were observed in patients receiving phototherapy. Comparing with previous studies, in Europe a study of 3,867 Scottish patients ${ }^{6}$, an increase risk of non-melanoma skin cancer among patients treated with both PUVA and NB-UVB was reported with 27 BCCs found compared with 14.1 expected in the matched population [6]. The risk of NMSC following long term NB-UVB and PUVA therapy has not been reported in Asian population. There was one report from Korea that had only one patient who developed basal cell carcinoma four months after NBUVB phototherapy which was not statistically difference between NB-UVB and control group [7]. The low incidence of non-melanoma skin cancer in our study and Korean study consistent with the previous study that 
explain by the effect of ethnicity on the risk of developing skin cancer [8]. Our negative study provide the important information for the patient who need long term phototherapy. Long term NB-UVB and PUVA therapy seem to be the safe therapeutics modalities for the patients with skin phototypes III-IV.

\section{Limitations}

This study was a retrospective study, so there could be some missing data and possible ascertainment bias.

\section{Conclusions}

This study did not show a significant increase in non-melanoma skin cancer risk among patients treated with long term PUVA and NB-UVB. The results suggest that PUVA and NB-UVB are safe modalities.

\section{References}

1. Vangipuram R, Feldman SR. Ultraviolet phototherapy for cutaneous diseases: a concise review. Oral Dis 7 (2015): 1-7.

2. Cummings SR, Tripp MK, Herrmann NB. Approaches to the prevention and control of skin cancer. Cancer Metastasis Rev 16 (1997): 309-327.

3. Stern R, Lunder E. Risk of squamous cell carcinoma and methoxsalen (psoralen) and UV-A radiation (PUVA): a metaanalysis. Arch Dermatol 134 (1998): 1582-1585.

4. Stern R, Lange R. Non-melanoma skin cancer occurring in patients treated with PUVA five to ten years after first treatment. J Invest Dermatol 91 (1988): 120-124.

5. Black RJ, Gavin AT. Photocarcinogenic risk of narrowband ultraviolet B (TL-01) phototherapy: early followup data. Br J Dermatol 154 (2006): 566-567.

6. Hearn R, Kerr A, Rahim K, Ferguson J, Dawe R. Incidence of skin cancers in 3867 patients treated with narrow-band ultraviolet B phototherapy. Br J Dermatol 159 (2008): 931-935.

7. Jo SJ, Kwon HH, Choi MR, Youn JI. No evidence for increased skin cancer risk in Koreans with skin phototypes III-V treated with narrowband UVB phototherapy. Acta Derm Venereol 91 (2011): 40-43.

8. Murase J, Lee E, Koo J. Effect of ethnicity on the risk of developing nonmelanoma skin cancer following long-term PUVA therapy. Int J Dermatol 44 (2004): 1016-1021.

9. Weischer M, Blum A, Eberhard F, et al. No evidence for increased skin cancer risk in psoriasis patients treated with broadband or narrowband UVB phototherapy: a first retrospective study. Acta Derm Venereol 84 (2004): 370-374.

This article is an open access article distributed under the terms and conditions of the

Creative Commons Attribution (CC-BY) license 4.0 